Hammersmith Hospital, Imperial College Healthcare NHS Trust, London, UK; ${ }^{3}$ Center of Pharmacology and Therapeutics, Imperial College London, London, UK

Introduction Better biomarkers are urgently needed to assist accurate diagnosis and appropriate treatment of malignant biliary obstruction, as, although malignancy is a common cause of obstructive jaundice, current diagnostic techniques often fail to differentiate benign from malignant disease. Molecular analysis of bile has recently produced promising candidate biomarkers. Previous work from our group found that biliary neutrophil gelatinase-associated lipocalin (NGAL), a small extracellular $25-\mathrm{kD}$ a protein with several biological functions, differentiates obstructive jaundice from malignancy from that in benign disease. The mechanism of NGAL in hepatopancreatobiliary (HPB) malignancy is unknown, although in other systems it promotes neoplastic diffusion by complexing and stabilising matrix metalloproteinase-9 (MMP9), enabling local invasion.

Aims (1) To investigate possible biliary complexing of MMP9 and NGAL as a mechanism of tumorigenesis. (2) To validate our previous findings of biliary NGAL as a novel biomarker of malignancy in biliary obstruction.

Methods Bile samples were collected from 77 patients undergoing ERCP ( $n=77,22$ with malignant disease and 55 with benign disease) at Imperial College London. ELISA was used to quantify levels of MMP9/NGAL complexes and of NGAL and MMP9 occurring independently in bile. Pearson's correlation analysis was used to determine the relationship between NGAL, MMP9 and NGAL/MMP9 complex levels, and statistical significance assessed by the Mann-Whitney U test.

Results Biliary NGAL levels were significantly higher in malignant biliary obstruction compared to benign disease (median $1555 \mathrm{ng} / \mathrm{ml}$ vs $402 \mathrm{ng} / \mathrm{ml}, \mathrm{p}=0.003$ ), giving a ROC AUC of 0.74 . Biliary MMP9 and NGAL/MMP9 complex levels were not different between these groups $(p=0.527, p=0.760)$. Unbound biliary NGAL and MMP9 levels correlated poorly $\left(r^{2}=0.03, p>0.05\right)$. Unbound NGAL correlated poorly with complex $\left(r^{2}=0.07, p>0.05\right)$ whereas unbound MMP9 correlated with NGAL/MMP9 complex level $\left(\mathrm{r}^{2}=0.73\right.$, $\mathrm{p}<0.05)$.

Conclusion This study is novel in confirming the presence of MMP9 in bile, alone and in complex with NGAL. However, although NGAL was increased in malignancy, MMP9 and MMP9/NGAL complex were not, suggesting that NGAL acts independently of MMP9 in endobiliary HPB malignancy. Mechanisms remain to be elucidated. This study also supports previous reports of NGAL as a novel and independent bile biomarker of malignant biliary obstruction.

Competing interests None declared.

\section{PTU-069 DETECTION OF CYSTIC DUCT STONES DURING LAPAROSCOPIC CHOLECYSTECTOMY}

\section{doi:10.1136/gutjnl-2012-302514c.69}

A Kambal, ${ }^{*}$ T Richards, H Jayman, Z Alsallami, T Lazim. General Surgery, Gwent Institute for Minimal Access Surgery, Newport, UK

Introduction With the advent of the Laparoscopic Cholecystectomy (LC) era, the loss of tactile element hindered the detection of cystic duct stones (CDS) during surgery. These stones are implicated in the post cholecystectomy pain syndrome, failure of the insertion of intra-operative cholangiogram (IOC) catheter and the subsequent development of common bile duct (CBD) stones. The aim of this analysis is to quantify the frequency of the incidental finding of CDS during LC.

Methods A cohort of consecutive patients undergoing LC during the period from November 2006 to May 2010 were included. Data were prospectively collected. Their liver function tests were documented in the preoperative period. The procedure entailed careful dissection of the cystic duct (CD) to the proximity of common bile duct. A clip was then placed at the gall bladder to CD junction. If an IOC was required, the $\mathrm{CD}$ was opened in the routine fashion. A partially closed endoclip was then used to milk the CD towards the gallbladder; any CDS encountered were retrieved and documented. If IOC was not indicated, the CD was milked prior to the application of gallbladder/CD clip.

Results The study included 330 patients; 80 male and 250 females. Age ranged between 16 and 88 years (Median 50, IOR: 36-62). In 266 patients no CDS were detected. However, in 64 (19\%) patients CDS were identified using the above technique; with 28 (45\%) having a single stone. The remaining $36(55 \%)$ patients had more than one stone with a maximum detected number of seven stones. Preoperative imaging failed to detect any CDS. Of those 64 patients with CDS, 47 (75\%) showed deranged liver function tests at some stage of their disease prior to surgery. In comparison, of the 266 patients with no CDS, 152 (57\%) also demonstrated abnormal liver function tests.

Conclusion The results demonstrate the fact pre-operative investigations are not helpful in diagnosing CDS. Their occurrence is common. In order to detect CDS, specific intra-operative awareness and vigilance are needed. Careful upward milking of the cystic duct before applying clips is a simple, safe and effective way of detecting and extracting these stones. This study changed our practice as this procedure is now included in all our Laparoscopic chlecystectomies.

Competing interests None declared.

\section{PTU-070 FINE MAPPING OF THE IL-2/IL-21 AND IL2RA LOCI IN PRIMARY SCLEROSING CHOLANGITIS}

doi:10.1136/gutjnl-2012-302514c.70

${ }^{1} \mathrm{~B}$ Srivastava, ${ }^{*}{ }^{1} \mathrm{G} \mathrm{F}$ Mells, ${ }^{2} \mathrm{H} \mathrm{J}$ Cordell, ${ }^{1} \mathrm{~A}$ Muriithi, ${ }^{1} \mathrm{M}$ Brown, ${ }^{3} \mathrm{E}$ Ellinghaus, ${ }^{3} \mathrm{~A}$ Franke, ${ }^{4} \mathrm{~T} \mathrm{H}$ Karlsen, ${ }^{1} \mathrm{R} N$ Sandford, ${ }^{5} \mathrm{G} \mathrm{J}$ Alexander, ${ }^{6} \mathrm{R} \mathrm{W}$ Chapman, ${ }^{7} \mathrm{~S}$ M Rushbrook, ${ }^{4} \mathrm{E}$ Melum. ${ }^{7}$ Academic Department of Medical Genetics, University of Cambridge, Cambridge, UK; ${ }^{2}$ Institute of Genetic Medicine, Newcastle University, Newcastle upon Tyne, UK; ${ }^{3}$ Institute of Clinical Molecular Biology, Christian-AlbrechtsUniversity, Kiel, Germany; ${ }^{4}$ Norwegian PSC Research Center, Oslo University Hospital, Rikshospitalet, Oslo, Norway; ${ }^{5}$ Department of Medicine, University of Cambridge, Cambridge, UK; ${ }^{6}$ Department of Hepatology, John Radcliffe University Hospitals NHS Trust, Oxford, UK; ${ }^{7}$ Department of Hepatology, Norfolk and Norwich University Hospitals NHS Trust, Norwich, UK

Introduction Recent genetic studies in Primary sclerosing cholangitis (PSC), a chronic bile duct disease, have shown suggestive association at IL-2/21 (4q27) and IL2RA (10p15). IL-2 and IL2RA are key regulators of immune tolerance. To further refine association at 4q27 and $10 \mathrm{p} 15$, a fine mapping study was undertaken in 1030 British PSC cases and 5162 healthy controls.

Methods For SNP selection, $80 \mathrm{Kbp}$ and $564 \mathrm{Kbp}$ regions were selected on 10p15 and 4q27, respectively, and SNP data from HapMap Data Rel 24/phase II was used to identify tag SNPs with Haploview v4.2. 62 tag SNPs were genotyped on a Sequenom platform. Control genotype data were available for 62 SNPs, previously genotyped in the Wellcome Trust Case Control Consortium 2 (WTCCC 2). 59 SNPs (28 at 4q27 and 31 at 10p15) passed quality control and were analysed using logistic regression in PLINK v1.07. For selected SNPs, previously published summary statistics ${ }^{1}$ were used to perform a meta-analysis.

Results Significant association $\left(\mathrm{p}<8.5 \times 10^{-4}\right)$ corrected for multiple testing (Bonferroni method) was observed for one SNP at $4 \mathrm{q} 27$ and three SNPs at 10p15 (Abstract PTU-070 table 1). In addition, nominal significance $(p<0.05)$ was seen for $9 / 27$ SNPs at $4 q 27$ and $10 / 28$ SNPs at $10 \mathrm{p} 15$. Genome-wide significance $\left(\mathrm{p}<5 \times 10^{-8}\right)$ was observed for rs4147359 (10p15) in the combined analysis. 